\title{
Intracranial Atherosclerosis in Stroke Patients, Which Risk Factors Are Important? A Study From Mixed Ethnic Population in Singapore.
}

shrikant Pande ( $\sim$ shrikantpande@yahoo.co.uk)

Changi General Hospital

May Thiri Lwin

Changi General Hospital

Aye Aye Khine

Changi General Hospital

May Win Myat

Changi General Hospital

Lorecar Lolong

Changi General Hospital

Julie Morris

University of Manchester

\section{Research Article}

Keywords: intracranial atherosclerotic disease, Asians, stroke, comorbidities of intracranial atherosclerosis

Posted Date: February 17th, 2021

DOI: https://doi.org/10.21203/rs.3.rs-199181/v1

License: (c) (i) This work is licensed under a Creative Commons Attribution 4.0 International License. Read Full License 


\section{Abstract}

Intracranial atherosclerosis (ICAD) although more common in Asians, has not been studied from Singapore population. The aim of this study is to identify prevalence ICAD in stroke subjects, its association with comorbidities, stroke subtypes and long-term survival. We performed retrospective analysis of 681 stroke patients, 327(48\%) had ICAD) with 232 (71\%) having one or more intracranial artery with significant stenosis. ICAD was associated with older age, ischaemic strokes, total anterior circulation syndrome (TACS), large artery strokes $(p<0.001)$, peripheral vascular disease (PVD, $p=0.018)$, diabetes mellitus (DM), and with history of hyperlipidemia. In addition, higher serum potassium $(p=0.046)$ and glucose $(p<0.001)$, lower haemoglobin $(p=0.040)$ and aortic valve sclerosis were significantly associated with ICAD $(\mathrm{p}<0.001)$.

Multivariable analysis showed a significant independent association of ICAD with advancing age, history of hyperlipidemia and DM.

In ischemic strokes (489), 311(64\%) had ICAD of which 229(72\%) had significant stenosis. Of the 192 hemorrhagic strokes, $16(8 \%)$ had ICAD.

Conclusion: The prevalence of ICAD, especially in ischemic strokes, from our study is high. As modifiable risk factors such as hyperlipidemia and DM appear to be associated with ICAD, then proactive management of these conditions may improve long term associated complications. Prospective studies may help to validate our findings.

\section{Introduction}

Intracranial and extracranial atherosclerosis have been associated with stroke. The prevalence of extracranial atherosclerosis is higher in Caucasian population and has been well studied. As compared to this, the risk of intracranial atherosclerotic disease (ICAD) in Asian population has been reported to be higher. Stroke associated with ICAD in Asian population has been reported between 33 to $56.3 \%$ as per previous studies. (1) and previous data from Singapore suggested $47.9 \%$ patients with stroke had ICAD. (2)

Due to advances in imaging technologies, an increasing number of patients are being diagnosed with ICAD.

Apart from stroke and recurrence of stroke, $(3,4,5)$, ICAD has been associated with increased risk of cognitive impairment(6), coronary artery disease(IHD).(7) Age, co-morbidities including metabolic syndrome, diabetes mellitus(DM), hypertension(HTN), dyslipidemia have been found to increase the risk of ICAD.(8)

Following acute ischemic stroke, the management of hypertension is crucial. However, in ischemic stroke patients with ICAD, lowering of blood pressure may worsen the ischaemic volume. (9) Considering these 
factors, it is important to understand the prevalence of ICAD and implement treatment strategies accordingly.

In the present study we aimed to estimate the prevalence of ICAD in patients diagnosed with stroke, identify the associated risk factors, and investigate its impact on long term survival.

\section{Methods}

Patients: This study was conducted in Changi general hospital, Singapore, with all the modern facilities for emergency and specialist care. The acute stroke unit is equipped with facilities for diagnosis and treatment including thrombolysis for strokes.

Management of stroke is streamlined through the emergency department, acute stroke unit, neurosurgical department, and if necessary, followed by transfer to the rehabilitation services. All the patients admitted with a diagnosis of stroke undergo the necessary investigations including those required to establish the underlying causes as per a standardised protocol based on established guidelines for stroke.

Based on the initial and subsequent clinical conditions and scan findings, the neurosurgical team orders further interventions. All the stroke patients are eventually referred to inpatient rehabilitation services. The neuro-rehabilitation department is equipped with all up-to date facilities. A multidisciplinary team review the patients throughout in-patient and their follow up as outpatients based on requirement.

This is a retrospective analysis of stroke patients (both infarction and spontaneous intracerebral haemorrhage) who had met the selection criteria of the study and were consecutively admitted to the neuro-rehabilitation facility at the Changi General Hospital from June 2008 to May 2017. The follow-up period ranged from 6 to 165 months. All the patients included in the current study were discharged from the rehabilitation facility and were followed up regularly as outpatients. The subsequent records of hospital admissions and follow up changes in the general physical and neurological status, and treatment regimens were available electronically and in paper format for all patients.

The exclusion criteria were (a) incomplete follow-up records including those patients who were repatriated to other countries, (b) patients less than 21 years of age (as per CIRB guideline), (c) transient ischaemic attacks.

Stroke and its subtypes were diagnosed by a stroke physician or neuro-surgeon on admission based on clinical examination, brain imaging [CT (computed tomography), MRI (magnetic resonance imaging), MRA (magnetic resonance angiography)], ECG (electrocardiography) 12 leads, continuous monitor or Holter, carotid Doppler and echocardiogram. The patients were classified as per Oxfordshire classification (for stroke territory) (10) and TOAST for ischaemic strokes. (11)

The Singhealth Centralized Institutional Review Board approved this study (2015/3112).. Informed consent from the patients was waived due to the retrospective nature of the study. All methods were performed in accordance with Singhealth CIRB guidelines approved for data collection, and storage. 
Sampling Procedure: All the electronic and paper medical records of the patients from the time stroke was diagnosed, follow up visits, and additional admissions were reviewed until October 2017. The demise date follow-up was until $21^{\text {st }}$ October 2019. The material was housed in the hospital's medical record database and in the records of the clinician at the neuro-rehabilitation facility. The data collected included demographic details, diagnosis, type of stroke (ischaemic, intracerebral bleed), and CT/MRI findings for stroke territory, admission electrolytes, clotting profiles, premorbid medications, and comorbidities. The treatment modalities included thrombolysis, medical treatments for raised intracranial pressure, neurosurgical interventions.

Statistical analysis: Categorical data are presented as frequency (percentage), and continuous data are presented as mean (standard deviation) for Normally distributed data and geometric mean and range for positively skewed data. Comparisons between patients with and without ICAD were made using twosample t-tests and logistic regression analysis, with odds ratios (OR) reported for the latter. Associations between mortality and ICAD were assessed using Cox proportional hazards regression. Hazard ratios (HR) and their associated $95 \%$ confidence intervals are presented.

A two-tailed $p$-value of $<0.05$ was considered to be statistically significant. The analysis was performed using the Statistical Package for the Social Sciences (SPSS) version 23.0 (IBM Corp. Armonk, New York).

\section{Results}

We analysed a total of 681 patients with stroke, of which 327(48\%) had ICAD. (Table1). Patients with advanced age, ischaemic strokes and with TACS were more likely to have ICAD $(p<0.001)$. Of all those with ICAD, $232(71 \%)$ had between one and seven intracranial arteries with significant stenosis. The imaging techniques used for diagnosis of stroke and ICAD are described. (Table 2)

Univariate analysis showed significant associations with ICAD for medical history of DM and hyperlipidaemia $(p<0.001)$, PVD $(p=0.018)$, and those patients receiving aspirin and lipid lowering agents $(p<0.001)$ premorbid. (Table 3$)$.

Admission blood parameters showed that higher levels of potassium $(p=0.046)$, glucose $(p<0.001)$ and lower HB $(p=0.040)$ were associated with ICAD. (Table 4$)$.

Patients with aortic valve sclerosis $(p<0.001)$ (Table 5) and ischaemic stroke patients with large artery strokes were more likely to have ICAD. (Table 6).

Multiple logistic regression analysis showed that the significant independent predictors of ICAD were advancing age $[\mathrm{OR}=1.03 ; 95 \% \mathrm{Cl}(1.01,1.04)]$, history of hyperlipidemia $[\mathrm{OR}=1.77(1.25,2.51)]$ and diabetes mellitus [OR=1.74 $(1.23,2.47)]$.

Survival and ICAD: Of the 327 patients with ICAD, 111 died during follow-up as compared to 104 of the 354 patients without ICAD. Patients with ICAD had significantly higher mortality; HR $=1.3295 \% \mathrm{Cl}(1.01$, 
1.73), $p=0.042$. However, this mortality difference was accounted for by the age disparity between those with and without ICAD [ age-adjusted HR=0.92 $(0.70,1.21), p=0.57$ ]. (Fig 1)

Subgroup analysis of ischemic and haemorrhagic strokes in relation to ICAD was carried out.

In 489 ischemic stroke patients, 311(64\%) patients had ICAD of which 229(72\%) had significant stenosis of one or more arteries. Age, TACS, hypertension, higher HDL and lower cholesterol /HDL ratio were associated with ICAD. Significant independent factors were higher admission HDL and lower cholesterol/HDL ratio.

Of the 192 patients with hemorrhagic stroke, 16 (8\%) had ICAD. Patients with DM, history of hyperlipidemia, higher levels of HDL and those on aspirin premorbid, had higher incidence of ICAD. Significant independent factors were a history of hyperlipidemia and high levels of HDL.

\section{Discussion}

In the present study the prevalence of ICAD is $48 \%$. We also noted higher prevalence of ICAD $(64 \%)$ in ischemic stroke patients along with stenosis $(72 \%)$ of intracranial arteries. Advancing age and TACS were associated with ICAD.

Previous studies have shown that stroke associated with ICAD in the Asian population is between 33 to $56.5 \%$ (1) and the data from Singapore suggested this figure of $47.9 \%$. (2)

Intracranial atherosclerosis may occur in isolation or associated with systemic atherosclerosis of peripheral arteries or coronaries. ICAD may be seen as irregularities in cerebral circulation or with varying degree of stenosis to complete occlusion.

Although ICAD can be diagnosed with noninvasive imaging techniques like transcranial doppler and MRA, the gold standard for the diagnosis is DSA. $(12,13,14,15)$

Autopsy studies are limited on ICAD and suggest that scattered calcifications (microscopic) are associated with cholesterol mediated intracranial large artery atherosclerosis, and stenosis as opposed to coalescent (macroscopic) calcifications

(16). Another study concluded that only intimal calcification as opposed to internal elastic lamina and adventitial calcification was associated with ICAD. (17)

Ma et al in their metanalysis concluded that age, metabolic syndrome, DM and HTN were risk factors for ICAD and that apolipoprotein A1 may offer protection against it.(8) ICAD also has been associated with extracranial carotid plaque burden as independent risk factor.(18)

Our findings are similar to previously published data in that patients with, DM, HTN and premorbid history of hyperlipidaemia, were more likely to have ICAD. 
We also noted higher serum glucose and potassium at the time of stroke showed association with ICAD. We are unsure whether these values are a result of stroke related complications and so need further evaluation.

The Hyderabad stroke registry sample of 2642 patients found $78.3 \%$ incidence of ICAD and $21.7 \%$ extracranial disease among the patients with large artery atherosclerosis (LAA) strokes. The authors noted association of ICAD with hyperlipidemia in LAA and those cardioembolic strokes.(19)

From our data those with large artery strokes had higher and those with lacunar infarcts had lower occurrence of ICAD.

Although the association of hyperlipidemia with ICAD has been demonstrated from past studies $(8,19$, 20 ), the APAC study concluded that triglycerides (TG) are a significant factor only for progression of atherosclerosis, (21) another study concluded TG: HDL cholesterol ratio to be significantly associated with ICAD in healthy subjects. (22)

We noted that ICAD was significantly associated in those with previous history of hyperlipidemia. The admission lipid profile in our study however suggested ICAD association with higher HDL and lower total cholesterol /HDL ratio.

Serum calcium has also been proposed as independent risk factor for ICAD.( 23)

From our study, only 221 patients provided data for calcium physiology. No significant association between serum calcium and ICAS was found, but this may be a result of the small number of patients in this group.

The relationship of haematocrit with ICAD is not well understood. From the Hisamaya study, where the authors prospectively studied population for 19 years and concluded that both high and low haematocrit levels were associated with ICAD. (24)

Our data suggests that low haematocrit values are associated with ICAD.

Biochemical markers such as higher levels of serum Aldosterone(25), homocysteine levels $(26,27)$ and 3 hydroxybutyrate (28) 18 have also been associated with development of ICAD.

PVD is a multisystem disease mostly associated with DM and smoking. We observed that those patients with PVD had increased incidence of ICAD, which may be a reflection of the associated comorbidities in these patients.

Literature search also suggested no association between ICAD and intracerebral bleed. (29). Our data of 192 patients with haemorrhagic stroke also shows a very low (8\%) incidence of ICAD.

Lee et.al concluded that ICAD presence prior to stroke and presence of large artery atherosclerosis may be related to development of early neurological deterioration following stroke. (30) Due to incomplete data 
on NIHSS or FIM scores, we are unable to draw any conclusion about these outcome measures in our study group.

Statins have been shown to be effective in patients with large artery atherosclerosis, as a result of decreased micro-embolic signal burden and better functional outcomes. (31) Statins have also shown to reduce the plaque enhancement and reduce large cortical lesions in patients with ICAD. (32) Other studies concluded better outcomes in neurological improvement, disability scores, survival, and stroke recurrence with statins. (33)

In the ARIC study however, the authors prospectively studied 14,175 asymptomatic populations over 2 years and found no association between the deranged lipid profile and ICAD. (34)

Our data suggest that patients with premorbid statin treatment until the time of stroke admission showed increased association with ICAD. Similarly, we also found that patients with premorbid treatment with aspirin had increased incidence of ICAD. However, these findings relate to univariate analysis. From the multivariable analysis which adjusted for age and comorbidities, only older patients, those with $D M$, and those with a history of hyperlipidemia were significantly more likely to have ICAD.

The association of aspirin and statin with ICAD from our study may be due to the interrelationships between factors which are related to ICAD i.e. aspirin users were older, and more likely to have ischaemic stroke and more likely to have DM compared to non-aspirin users. Therefore, the association between aspirin, statin use and ICAD may be explained by confounding factors.

Chronic kidney disease (CKD) and end stage renal failure (ESRF) leads to increased calcification, especially in the intracranial arteries. This results in altered autoregulation and increased risk of cerebrovascular events in this group of people, especially those undergoing haemodialysis.

In our study, patients with normal renal function, those with acute kidney injury, chronic kidney disease and end stage renal failure as per KEDIGO classification were analysed. (35) However, there was no correlation between impaired renal function and ICAD. This is likely due to the small number of patients in the ESRF and CKD groups.

As a part of the stroke work-up, patients undergo echocardiography. We analysed the findings on echocardiography with ICAD. We concluded that patients with ICAD had a significant association with aortic sclerosis. This could be related to both of these conditions with advancing age.

Treatment strategies: From the WASID trial, warfarin did not show an advantage as compared to Aspirin in relation to ischemic strokes, intracerebral haemorrhage or vascular deaths.(36)

CHANCE subgroup analysis concluded that the combination of aspirin and clopidogrel in patients with ICAD was not different as compared to monotherapy, for reduction of recurrent stroke risk.(37) 
The results of the SAMMPRIS trial concluded that "aggressive medical treatment as a combination of aspirin and clopidogrel was superior to stenting". (38)

The sub-study of the CHANCE trial found that aspirin and clopidogrel combination therapy did not reduce the rate of new strokes in patients with carrier of CYP2C19 gene variants. As this gene variant is more prevalent in Asians than whites, (39) and the prevalence of ICAD is more in the same group of patients, alternative strategies for treatment of ICAD are necessary.(39)

Cilostazol is a vasodilating, anti-inflammatory and anti-atherogenic agent and has been studied in TOSS 1 and 2 trial. It has shown benefits in terms of reducing progression of ICAS and change in stenosis of atherosclerotic vessel. $(40,41)$

Both the WASID and TOSS 2 trials indicated very high BP, as systolic readings above $160 \mathrm{~mm}$ of $\mathrm{Hg}$, were associated with increased risk of recurrent strokes and ICAD progression.

Early neurological deterioration has been shown with antihypertensive use within 7 days of stroke in patients with symptomatic large artery stenosis.(42)

Although lipid-lowering treatment is recommended for reduction of stroke risk, its role in ICAD is currently not clear.(43)

Small studies have demonstrated that statins were useful in prevention of ICAD progression, (44) and similar results were observed in high dose statin treatment group from Chinese study. (45)

Conclusion: It appears from previous literature and our study that incidence of ICAD is significant in the Asian population. Whether ICAD is part of systemic atherosclerosis associated with ageing, needs further investigation. Proactive management in this group of patients for the modifiable risk factors including DM and Hyperlipidemia are important. Clopidogrel resistance in Chinese ethnicity warrants alternative treatment modalities in these group of patients.

The percentage of ICAD could be even higher with application of DSA which is a gold standard for diagnosis.

Strengths of our study: probably the first data on intracranial atherosclerosis from Singapore comparing comorbidities, laboratory parameters and long term survival.

We also reviewed the differences between ischemic and haemorrhagic strokes.

The co-morbidities like peripheral vascular disease and association with echocardiographic findings such as aortic sclerosis, mitral valve calcifications, which have not been well studied before.

Limitations: those patients with mild strokes and TIA are not represented in the study.

If all patients were investigated with CT angiography or DSA, the ICAD incidence could be even higher. 
Survival data is compared to other cardiovascular comorbidities and hence may not be a true reflection.

\section{Abbreviations}

TACS: total anterior circulation syndrome, PACS: partial anterior circulation syndrome, LACS: lacunar syndrome, POCS: posterior circulations syndrome.

\section{Declarations}

Author's contributions: All the authors have contributed to the study, me Pande

Pande S.D., Lwin M. T., Khine A A, Win M M, Lolong L. with data collection,

literature searches and manuscript preparation, Morris $\mathrm{J}$, with statistical analysis.

Declaration: We confirm that we have read the journal's position on issues involved in ethical publication and affirm that this report is consistent with those guidelines, ICMJE criteria are met.

Consent for data on human subjects: SingHealth CIRB (2015/3112) approved our study for data collection, study protocol conforms to the ethical guidelines of the 1975 Declaration of Helsinki and waiver of consent was obtained due to retrospective nature of the study.

Consent for publication: SingHealth IRB.

Availability of data: we will seek SingHealth IRB approval for this.

Competing interest: none declared.

Funding: No funding was obtained from any source for this study.

\section{References}

1. Wong, L. K. S. Global burden of intracranial atherosclerosis.Int J Stroke.2006Aug;1(3):158-9.

2. Chang, H. M., Gan, H. Y., Lee, M. P., Wong, M. C. \& Chen, C. P. Intracranial disease in Singaporean stroke patients. 10th European Stroke Conference 2000. Lisbon.

3. Wong, K. S. \& Li, H. Long-term mortality, and recurrent stroke risk among Chinese stroke patients with predominant intracranial atherosclerosis. Stroke. 34, 2362-2366 (2003).

4. Mazighi, M. et al. Prospective study of symptomatic atherothrombotic intracranial stenosis. The GESICA study. Neurology. 66, 1187-1191 (2006).

5. Kasner, S. E. et al. et.al. Predictors of ischemic stroke in the territory of symptomatic intracranial arterial stenosis. Circulation. 113, 555-563 (2006).

6. Dearborn, J. L. et al. et.al. Intracranial atherosclerosis and dementia: The Atherosclerosis Risk in Communities (ARIC) Study. Neurology.2017 Apr 18;88(16):1556-1563. 
7. Oh, H. G., Chung, P. W. \& Rhee, E. J. Increased risk for intracranial arterial stenosis in subjects with coronary artery calcification. Stroke. 46 (1), 151-156 (2015 Jan).

8. Ma, Y. H. et al. et.al. Risk factors for intracranial atherosclerosis: A systematic review and metaanalysis.Atherosclerosis.2019 Feb;281:71-77.

9. Park, J. M. et al. Intensive blood pressure control may not be safe in subacute ischemic stroke by intracranial atherosclerosis: a result of randomized trial. J Hypertens. 36 (9), 1936-1941 (2018 Sep).

10. Bamford, J., Sandercock, P., Dennis, M., Burn, J. \& Warlow, C. Classification, and natural history of clinically identifiable subtypes of cerebral infarction. Lancet. 337, 1521-1526 (1991).

11. Adams, H. P. et al. et.al. Classification of subtype of acute ischemic stroke. Definitions for use in a multicenter clinical trial. TOAST. Trial of Org 10172 in Acute Stroke

Treatment.Stroke.1993Jan;24(1):35-41.

12. Babikian, V. L., Feldmann, E. \& Wechsler, L. R. Transcranial Doppler Ultrasonography: year 2000 update. Neuroimaging. 10, 101-115 (2000).

13. Wong, K. S. et al. Variability of magnetic resonance angiography and computed tomography angiography in grading middle cerebral artery stenosis. Stroke. 27, 1084-1087 (1996).

14. Suwanwela, N. C., Phanthumchinda, K. \& Suwanwela, N. Transcranial Doppler sonography and CT angiography in patients with atherothrombotic middle cerebral artery stroke. Am J Neuroradiol. 23, 1352-1355 (2002).

15. Navarro, J. C., Mikulik, R., Garami, Z. \& Alexandrov, A. V. The accuracy of transcranial Doppler in the diagnosis of stenosis or occlusion of the terminal internal carotid artery. J Neuroimaging. 14, 314318 (2004).

16. Shapiro, S. D. et al. et.al. Pathological correlates of brain arterial calcifications. Cardiovasc Pathol.2019 Jan-Feb;38;7-13.

17. Yang, W. J. et al. et.al. Postmortem Study Exploring Distribution and Pattern of Intracranial Artery Calcification.Stroke.2018Nov;49(11):2767-2769.

18. Xu, Y. et al. et.al. Association of severity between carotids and intracranial artery atherosclerosis. Ann Clin Transl Neurol. Jun4;5 (7), 843-849 (2018).

19. Kaul, S. et al. et.al Intracranial Atherosclerosis is the most Common Stroke Subtype: Ten-year Data from Hyderabad Stroke Registry (India). Ann Indian Acad Neurol. 21 (3), 209-213 (2018 Jul-Sep).

20. Suri, M. F. \& Johnston, S. C. Epidemiology of intracranial stenosis. J Neuroimaging. 19 (Suppl 1), $11-$ 16 (2009 Oct).

21. Wu, J., Wang, Y., Wang, A., Xie, J. \& Zhao, X. Association between fasting Triglyceride levels and the Prevalence of Asymptomatic Intracranial Arterial Stenosis in a Chinese Community-based Study. Sci Rep.2019 Apr10:8(1):5744.

22. Kang, K., Lee, K. \& Chung, S. H. The triglyceride: high-density lipoprotein-cholesterol ratio and stenoocclusive disease in the intracranial arteries. J Thromb Thrombolysis. 32 (1), 103-109 (2011 Jul). 
23. Ishizaka, N. et al. et.al. Serum calcium concentration and carotid artery plaque: a population-based study. Cardiol. 39 (3), 151-157 (2002. Mar).

24. Gotoh, S. et al. et.al. Haematocrit and the risk of cardiovascular disease in a Japanese community: The Hisamaya Study. Atherosclerosis. 242 (1), 199-204 (2015 Sep).

25. Zhang, S. et al. et.al. Serum Aldosterone Is Associated with Cerebral Artery Atherosclerosis and Calcification. J Stroke Cerebrovasc Dis 2019 MAR;28(3):523-530.

26. Gungor, L., Polat, M., Ozberk, M. B., Avci, B. \& Abur, U. Which Ischemic Stroke Subtype Is Associated with Hyperhomocysteinemia?J Stroke Cerebrovasc Dis..2018 Jul;27(7):1921-1929.

27. Lu, S. S. et al. Plasma Homocysteine levels and intracranial plaque characteristics: association and clinical relevance in ischemic stroke. BMC Neurol. 6 (1), 200 (2018 Dec).

28. Vojinovic, D. et al. et.al. Metabolic profiling of intra-and extracranial atherosclerosis.Atherosclerosis.2018 May;272:60-65.

29. Bouloiis, G. et al. et.al. Intracranial atherosclerosis and cerebral small vessel disease in intracerebral haemorrhage patients. J Neurol Sci. 15, 369324-369329 (2016 Oct).

30. Lee, S. J. \& Lee, D. G. Distribution of atherosclerotic stenosis determining early neurologic deterioration in acute ischemic stroke.PloS One.2017 Sep25;12(9).

31. Safouris, A. et al. et.al. Statin Pretreatment and Micro embolic Signals in Large Artery Atherosclerosis. Arterioscler Thromb Vasc Bio. 37 (7), 1415-1422 (2017 Jul).

32. Chung, J. W., Hwang, J., Lee, M. J., Cha, J. \& Bang, C. Y. Previous Stain Use and High-Resolution Magnetic Resonance Imaging Characteristics of Intracranial Atherosclerotic Plaque: The Intensive Statin Treatment in Acute Ischemic Stroke Patients With Intracranial Atherosclerosis Study. Stroke. 47 (7), 1789-1796 (2016 Jul).

33. Denswil, N. P. et al. et.al. Atherosclerosis in the circle of Willis: Spatial differences in composition and in distribution of plaques. Atherosclerosis. 251, 78-84 (2016 Aug).

34. Shahar, E. et al. et.al. Plasma lipid profile and incident ischemic stroke: The Atherosclerosis Risk in Communities (ARIC) study. Stroke. 34 (3), 623-631 (2003 Mar).

35. KEDIGO 2012 Clinical Practice Guideline for the Evaluation and Management of Chronic Kidney Disease. Kidney Int Supple 2013; 3:1-150.

36. Chimowitz, M. I. et al. et.al. Comparison of warfarin and aspirin for symptomatic intracranial arterial stenosis. N Eng J Med. 352, 1305-1306 (2005).

37. Wang, Y. et al. et.al. Clopidogrel with aspirin in acute minor stroke or transient ischemic attack. N Eng J Med. 369, 11-19 (2013).

38. Chimowitz, M. I. et al. et.al. Stenting versus aggressive medical therapy for intracranial arterial stenosis. N Eng J Med. 365, 993-1003 (2011).

39. Wang, Y. et al. et.al. Association between cyp2c19 loss-of-function allele status and efficacy of clopidogrel for risk reduction among patients with minor stroke or transient ischemic attack. JAMA. 316, 70-78 (2016). 
40. Kwon, S. U. et al. et.al. Cilostazol prevents the progression of the symptomatic intracranial arterial stenosis: the multicenter double-blind placebo-controlled trial of cilostazol in symptomatic intracranial arterial stenosis. Stroke. 36, 782-786 (2005).

41. Kwon, S. U. et al. et.al. Efficacy and safety of combination antiplatelet therapies in patients with symptomatic intracranial atherosclerotic stenosis. Stroke. 42, 2883-2890 (2011).

42. Oh, M. S. et al. et.al. Modest blood pressure reduction with valsartan in acute ischemic stroke: a prospective, randomized, open-label, blinded-end-point trial. Int J Stroke. 10, 745-751 (2015).

43. Kim, J. S. \& Bang, O. Y. Medical Treatment if Intracranial Atherosclerosis: An Update. Journal of Stroke. 19 (3), 261-270 (2017).

44. Kim, H. J., Kim, E. K., Kwon, S. U., Kim, J. S. \& Kang, D. W. Effect of statin on progression of symptomatic intracranial atherosclerosis. Can J Neurolo Sci. 39, 801-806 (2012).

45. Zhou, P. et al. et.al. Efficacy and safety of intensive statin therapy in Chinese patients with atherosclerotic intracranial arterial stenosis: a single-center, randomized, single-blind, parallel-group study with one-year follow-up.Clin Neurol Neurosurg2014;1206-13.

\section{Tables}

Table 1: Demographic and clinical characteristics of the patients in relation to ICAD. 


\begin{tabular}{|c|c|c|c|c|}
\hline- & - & ICAD & & \\
\hline \multirow[t]{3}{*}{ - } & - & No & Yes & p-value \\
\hline & & $(n=354)$ & $(n=327)$ & \\
\hline & & & - & - \\
\hline \multirow[t]{2}{*}{ Gender } & Male $(n=423)$ & $54 \%$ & $46 \%$ & 0.16 \\
\hline & Female $(\mathrm{n}=258)$ & $48 \%$ & $52 \%$ & \\
\hline- & - & - & & \\
\hline \multirow[t]{4}{*}{ Race } & Chinese $(n=447)$ & $54 \%$ & $46 \%$ & 0.22 \\
\hline & Indian $(n=55)$ & $44 \%$ & $56 \%$ & \\
\hline & Malay $(n=152)$ & $53 \%$ & $47 \%$ & \\
\hline & Others $(n=27)$ & $37 \%$ & $63 \%$ & \\
\hline \multicolumn{5}{|l|}{ Age, mean (SD) } \\
\hline & & $61.8(13.4)$ & $66.8(11.7)$ & $<0.001$ * \\
\hline \multirow[t]{2}{*}{ Cerebral Bleed } & Ischaemic stroke $(n=489)$ & $36 \%$ & $64 \%$ & $<0.001$ * \\
\hline & Intracerebral Bleed $(n=192)$ & $92 \%$ & $8 \%$ & \\
\hline \multirow[t]{4}{*}{ Cardio-embolic stroke risk } & None $(n=288)$ & $52 \%$ & $48 \%$ & 0.090 \\
\hline & Moderate risk $(n=73)$ & $38 \%$ & $62 \%$ & \\
\hline & High risk $(n=185)$ & $48 \%$ & $52 \%$ & \\
\hline & Not done $(n=35)$ & & & \\
\hline \multirow[t]{5}{*}{ Distribution of stroke territory } & TACS $(n=30)$ & $40 \%$ & $60 \%$ & $<0.001$ * \\
\hline & PACS $(n=187)$ & $43 \%$ & $57 \%$ & \\
\hline & LACS $(n=323)$ & $63 \%$ & $37 \%$ & \\
\hline & POCS (n=131) & $43 \%$ & $57 \%$ & \\
\hline & Unidentified $(n=10)$ & & & \\
\hline
\end{tabular}


Table 2: Imaging techniques performed for diagnosis of ICAD

\begin{tabular}{|ll|}
\hline Imaging technique & Number of stroke patients \\
\hline CT & 246 \\
\hline MRI & 504 \\
\hline MRA & 17 \\
\hline CT angiography & 71 \\
\hline
\end{tabular}

CT: Computed tomography. MRI: Magnetic resonance imaging, MRA; Magnetic resonance angiogram, CT angiography: Computed tomography angiogram.

Note: Patients could have undergone more than one technique.

Table 3: Clinical characteristics in relation to ICAD 


\begin{tabular}{|c|c|c|c|c|}
\hline - & _ & ICAD & & \\
\hline - & - & No & Yes & $\mathrm{p}-$ \\
\hline & & $(n=354)$ & $(n=327)$ & \\
\hline & & & - & - \\
\hline Atrial fibrillation & No $(n=506)$ & $53 \%$ & $47 \%$ & 0.66 \\
\hline & Yes $(n=175)$ & $50 \%$ & $50 \%$ & \\
\hline- & - & - & & \\
\hline Peripheral & No $(n=600)$ & $54 \%$ & $46 \%$ & 0.018 \\
\hline & Yes $(n=81)$ & $40 \%$ & $60 \%$ & \\
\hline Hypertension & No $(n=168)$ & $54 \%$ & $46 \%$ & 0.66 \\
\hline & Yes $(n=513)$ & $52 \%$ & $48 \%$ & \\
\hline Diabetes mellitus & No $(n=412)$ & $60 \%$ & $40 \%$ & $\begin{array}{l}< \\
0 \\
\star\end{array}$ \\
\hline & Yes $(n=269)$ & $40 \%$ & $60 \%$ & \\
\hline Hyperlipidaemia & No $(n=383)$ & $62 \%$ & $38 \%$ & $\begin{array}{l}< \\
\underset{*}{*} .001\end{array}$ \\
\hline & Yes $(n=298)$ & $39 \%$ & $61 \%$ & \\
\hline Renal dysfunction & Normal $(n=494)$ & $54 \%$ & $46 \%$ & 0.20 \\
\hline & Acute kidney injury ( $n=39)$ & $38 \%$ & $62 \%$ & \\
\hline & Chronic kidney disease $(n=32)$ & $56 \%$ & $44 \%$ & \\
\hline & $\begin{array}{l}\text { Acute later progressed to chronic kidney } \\
\text { disease }(n=9)\end{array}$ & $78 \%$ & $22 \%$ & \\
\hline & $\begin{array}{l}\text { Normal renal function progressed to chronic } \\
\text { kidney disease }(n=67)\end{array}$ & $45 \%$ & $55 \%$ & \\
\hline & $\begin{array}{l}\text { Chronic kidney disease later progressed to end- } \\
\qquad \text { Page } 15 / 19\end{array}$ & $44 \%$ & $56 \%$ & \\
\hline
\end{tabular}


stage renal failure $(n=25)$

end-stage renal failure $(n=8)$

$38 \% \quad 62 \%$

Normal function to end-stage renal failure $(n=\quad 71 \% \quad 29 \%$

7)

\begin{tabular}{|c|c|c|c|c|}
\hline Aspirin & No $(n=512)$ & $56 \%$ & $44 \%$ & $\begin{array}{l}< \\
0.001\end{array}$ \\
\hline & Yes $(n=140)$ & $44 \%$ & $56 \%$ & \\
\hline & Dual anti-platelets $(n=23)$ & $17 \%$ & $83 \%$ & \\
\hline Statin & No $(n=436)$ & $58 \%$ & $42 \%$ & $\begin{array}{l}< \\
\underset{*}{0} .001\end{array}$ \\
\hline & Statin $(n=213)$ & $40 \%$ & $60 \%$ & \\
\hline & Fibrate $(n=9)$ & $67 \%$ & $33 \%$ & \\
\hline & Both $(n=23)$ & $35 \%$ & $65 \%$ & \\
\hline Warfarin & No $(n=654)$ & $52 \%$ & $48 \%$ & 0.33 \\
\hline & Yes $(n=27)$ & $63 \%$ & $37 \%$ & \\
\hline
\end{tabular}

Table 4: Admission bloods and ICAD 


\begin{tabular}{|c|c|c|c|}
\hline - & mean $(S D) ;$ & nge & \\
\hline - & No ICAD & ICAD & $\mathrm{p}$-value \\
\hline & $(n=354)$ & $(n=327)$ & \\
\hline Cholesterol/High density lipoprotein ratio & 4.84 (1.79). & 4.74 (1.64). & 0.42 \\
\hline$(n=324: 326)^{a}$ & $0.39,13.3$ & $0.34,10.2$ & \\
\hline Total cholesterol & $5.26(1.41)$ & $5.39(1.37)$ & 0.24 \\
\hline$(n=324: 326)^{a}$ & $0.7,10.8$ & $2.3,10.2$ & \\
\hline High density lipoprotein $(n=324: 326)^{a}$ & 1.17 (0.38). & $1.26(0.70)$ & 0.07 \\
\hline & $0.4,3.6$ & $0.6,10.5$ & \\
\hline Triglycerides ${ }^{b}$ & 1.26. & 1.29 . & 0.61 \\
\hline$(n=324: 325)^{a}$ & $0.2,8.1$ & $0.4,7.1$ & \\
\hline Low density lipoprotein cholesterol & 3.45 (1.24). & $3.53(1.26)$. & 0.45 \\
\hline$(n=316: 324)^{a}$ & $0.9,8.3$ & $0.8,7.3$ & \\
\hline Urea ${ }^{b}$ & 4.68. & 4.89. & 0.20 \\
\hline$(n=352: 326)^{a}$ & $1.1,26.8$ & $1.4,24.4$ & \\
\hline Sodium & 137.7 (3.4). & $137.4(3.5)$ & 0.16 \\
\hline & 120,147 & 118,145 & \\
\hline Potassium & $3.98(0.70)$. & $4.08(0.62)$. & 0.046 * \\
\hline & $2.0,8.5$ & $2.8,7.3$ & \\
\hline Glucose ${ }^{b}$ & 8.35. & 9.44 & $<0.001$ \\
\hline & $4.3,27$ & $4.7,48$ & \\
\hline Creatinine $^{a}$ & 87.4. & 90.4 & 0.36 \\
\hline & 31,752 & 33,581 & \\
\hline Creatinine category & & & 0.64 \\
\hline$(n=22)$ Low $<50$ & $46 \%$ & $54 \%$ & \\
\hline$(n=386)$ Normal 50-90 & $53 \%$ & $47 \%$ & \\
\hline$(n=273)$ High $>90$ & $50 \%$ & $50 \%$ & \\
\hline Haemoglobin ${ }^{b}$ & 14.1 & 13.8. & 0.040 * \\
\hline
\end{tabular}




\begin{tabular}{|llll|}
\hline White blood cell count & $9,47.4$ & $6.4,19.6$ & \\
& $10.1(3.7)$. & $9.6(3.1)$. & 0.08 \\
\hline Platelets & $3.5,27.7$ & $4.1,24.5$ & \\
$(\mathrm{n}=354: 326)^{a}$ & $283(97)$. & $276(82)$. & 0.36 \\
\hline Calcium & 85,820 & 87,655 & \\
$(\mathrm{n}=221: 224)^{a}$ & $2.27(0.13)$. & $2.28(0.10)$. & 0.12 \\
\hline Phosphate & $1.03,2.67$ & $2.04,2.74$ & \\
$(\mathrm{n}=222: 224)^{a}$ & $1.22(0.33)$. & $1.18(0.27)$. & 0.21 \\
\hline
\end{tabular}

a: subgroup numbers for measure with missing data

b: Data followed a highly skewed distribution and were loge transformed prior to analysis. Geometric means and ranges are presented for these variables

Table 5: Echocardiography abnormalities in relation to ICAD

\begin{tabular}{|c|c|c|c|c|}
\hline - & - & ICAD & & \\
\hline \multirow[t]{2}{*}{ - } & - & $\begin{array}{l}\text { No } \\
(n=354)\end{array}$ & $\begin{array}{l}\text { Yes } \\
(n=327)\end{array}$ & $\mathrm{p}$-value \\
\hline & & & - & - \\
\hline \multirow[t]{2}{*}{ Mitral annular calcification } & No $(n=613)$ & $53 \%$ & $47 \%$ & 0.16 \\
\hline & Yes $(n=56)$ & $43 \%$ & $57 \%$ & \\
\hline- & - & - & & \\
\hline \multirow[t]{2}{*}{ Mitral valve calcification } & No $(n=656)$ & $52 \%$ & $48 \%$ & 0.24 \\
\hline & Yes $(n=19)$ & $37 \%$ & $63 \%$ & \\
\hline \multirow[t]{3}{*}{ Aortic valve calcification } & No $(n=422)$ & $58 \%$ & $42 \%$ & $<0.001$ * \\
\hline & $\operatorname{AVSCL}(n=248)$ & $41 \%$ & $59 \%$ & \\
\hline & Degenerative stenosis $(n=8)$ & $38 \%$ & $62 \%$ & \\
\hline
\end{tabular}

Table 6: Ischaemic stroke patients: characteristics by ICAD 


\begin{tabular}{|lllll|}
\hline- & - & ICAD & & \\
\hline- & - & No & Yes & p-value \\
& & $(n=178)$ & $(n=311)$ & \\
\hline Artery & Small $(n=187)$ & $44 \%$ & $56 \%$ & $<0.001$ * \\
\hline & Large $(n=299)$ & $31 \%$ & $69 \%$ & \\
\hline Others $(n=3)$ & $67 \%$ & $33 \%$ & \\
\hline
\end{tabular}

Figures

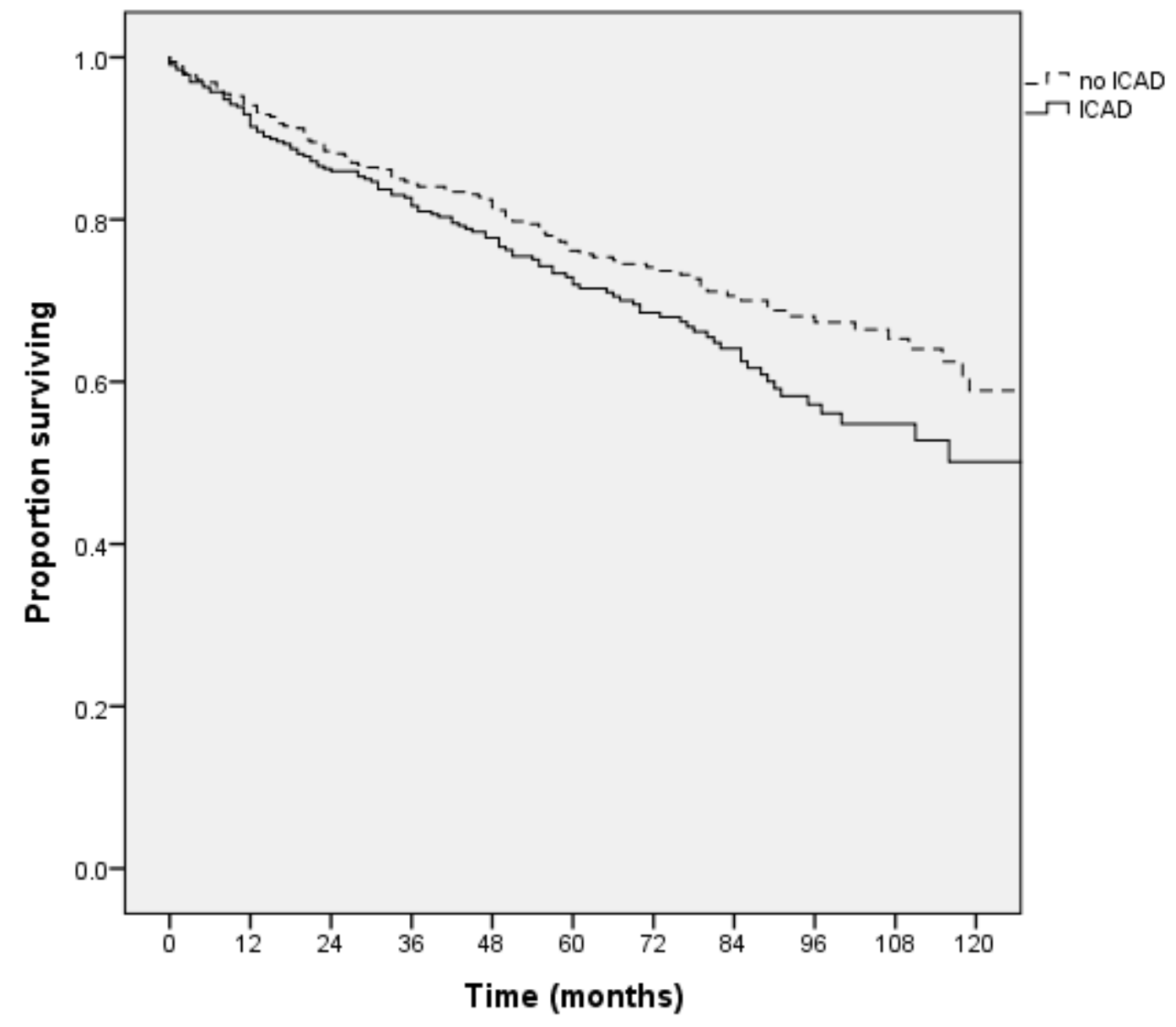

Figure 1

Although the patients with ICAD had significantly higher mortality; $[\mathrm{HR}=1.3295 \% \mathrm{Cl}(1.01,1.73)$, $p=0.042]$, the age-adjusted survival $(H R=0.92(0.70,1.21), p=0.57)$ was not significant. 